J Cell Sci. 2005;118(pt 23):5489-5498.

12. Jiang JG, et al. Cytochrome p450 epoxygenase promotes human cancer metastasis. Cancer Res. 2007; 67(14):6665-6674.

13. Chen C, et al. Selective inhibitors of CYP2J2 related to terfenadine exhibit strong activity against human cancers in vitro and in vivo.J Pharmacol Exp Ther. 2009;329(3):908-918.

14. Homma S, et al. Antigenic stimulation with cytochrome P450 2J expressed in mouse hepatocellular carcinoma cells regulates host anti-tumour immunity. Clin Exp Immunol. 2009;156(2):344-352.

15. Zagorac D, Jakovcevic D, Gebremedhin D, Harder DR. Antiangiogenic effect of inhibitors of cytochrome $\mathrm{P} 450$ on rats with glioblas- toma multiforme. J Cereb Blood Flow Metab. 2008; 28(8):1431-1439.

16. Nithipatikom K, et al. Inhibition of carcinoma cell motility by epoxyeicosatrienoicacid(EET) antagonists. Cancer Sci. 2010;101(12):2629-2636.

17. Spector AA. Arachidonic acid cytochrome P450 epoxygenase pathway. J Lipid Res. 2009;50(suppl):S52-S56.

18 . Yang W, et al. Characterization of 14,15 -epoxyeicosatrienoyl-sulfonamides as 14,15-epoxyeicosatrienoic acid agonists: use for studies of metabolism and ligand binding. J Pharmacol Exp Ther. 2007; 321(3):1023-1031.

19. Liu Y, et al. The antiinflammatory effect of laminar flow: the role of PPARgamma, epoxyeicosatrienoic acids, and soluble epoxide hydrolase. Proc Natl Acad
Sci U S A. 2005;102(46):16747-16752.

20. Fang X, et al. 14,15-Dihydroxyeicosatrienoic acid activates peroxisome proliferator-activated receptor-alpha. Am J Physiol Heart Circ Physiol. 2006; 290(1):H55-H63.

21. Cheng LM, et al. The epoxyeicosatrienoic acidstimulated phosphorylation of EGF-R involves the activation of metalloproteinases and the release of HB-EGF in cancer cells. Acta Pharmacol Sin. 2010; 31(2):211-218

22. Liu L, et al. Epoxyeicosatrienoic acids attenuate reactive oxygen species level, mitochondrial dysfunction, caspase activation, and apoptosis in carcinoma cells treated with arsenic trioxide.J Pharmacol Exp Ther. 2011;339(2):451-463.

\title{
Alteration of hypothalamic cellular dynamics in obesity
}

\author{
Edward B. Lee ${ }^{1}$ and Rexford S. Ahima ${ }^{2}$
}

\begin{abstract}
${ }^{1}$ Department of Pathology and Laboratory Medicine and 2Department of Medicine, Division of Endocrinology, Diabetes and Metabolism, Perelman School of Medicine at the University of Pennsylvania, Philadelphia, Pennsylvania, USA.
\end{abstract}

\begin{abstract}
The number of people who suffer from obesity and one or more of its adverse complications is rapidly increasing. It is becoming clear that diet, exercise, and other lifestyle modifications are insufficient strategies to combat this growing problem. Greater understanding of the mechanisms controlling our desire to feed and our ability to balance energy intake with energy expenditure are key to the development of pharmacological approaches for treating obesity. Although great strides have been made in our understanding of how the hypothalamus regulates feeding and energy balance, much less is known about how obesity affects the structure of the hypothalamus. The authors of two papers in this issue of the JCI have addressed this issue by examining the effects of obesity on neurons and glia in the hypothalamus. These studies reveal that obesity may be in part due to hypothalamic injury, which leads to inflammation and reduced neurogenesis. These findings support the notion that obesity is a disease that affects multiple organs, including the brain, and that disruption of normal brain function leads to abnormal regulation of peripheral metabolism.
\end{abstract}

\section{Introduction}

As the number of people who are obese continues to rise so does the number of cases of obesity-associated morbidities, including type 2 diabetes, hypertension, heart disease, sleep apnea, and cancer. Diet, exercise, and other lifestyle modifications are important approaches to treating obesity. However, it is becoming clear that pharmacological approaches are also needed to combat this major healthcare problem. Thus far, pharmacological approaches to treating obesity have shown limited efficacy in the clinic and have been associated with serious side

Conflict of interest: The authors have declared that no conflict of interest exists.

Citation for this article: J Clin Invest. 2012; 122(1):22-25. doi:10.1172/JCI61562. effects. This is largely because our understanding of the complex biology of obesity is incomplete. For example, while great strides have been made in our understanding of how the brain controls our desire to feed, as well as the processes underlying the balancing of energy intake and expenditure, little is known about how the structure and organization of the brain are altered by obesity.

\section{Central regulation of peripheral metabolism}

The role of the hypothalamus in the regulation of feeding and energy balance was first highlighted by lesion studies in rodents ( 1 , 2 ). Although these classic studies proposing the existence of "feeding" and "satiety" centers in the hypothalamus lacked anatomic precision and were overly simplistic in their interpretation, the importance of the hypothalamus in the regulation of feeding and energy balance was subsequently highlighted by the discovery that hormone and peptide regulators of feeding and metabolism act on the hypothalamus as well as the brainstem and other areas of the brain (3). The adipose tissue-derived hormone leptin exerts its inhibitory effects on food intake by modulating the function of neurons in the arcuate nucleus (an aggregation of neurons in the medial basal region of the hypothalamus). Specifically, leptin suppresses neurons that release neuropeptide Y (NPY) and agouti-related peptide - neuropeptides that normally increase appetite - while stimulating neurons that release proopiomelanocortin (POMC) - the precursor of several neuropeptides, including some that suppress appetite (3). Leptin, ghrelin, and other hormones that control energy metabolism also affect synaptic plasticity - the structural connections of neurons in the hypothalamus and other areas of the brain (4-7).

A hallmark of obesity is the ability of adipose tissue to expand and undergo significant remodeling in order to fulfill its role as the major energy-storing organ. This involves coordinated responses among various cell types, including adipocyte precursors, blood vessels, and immune cells (8). Inflammation in obese adipose tissue is related to adipocyte death, accumulation of macrophages and other immune cells, and metabolic dysfunction (8). Recent stud- 


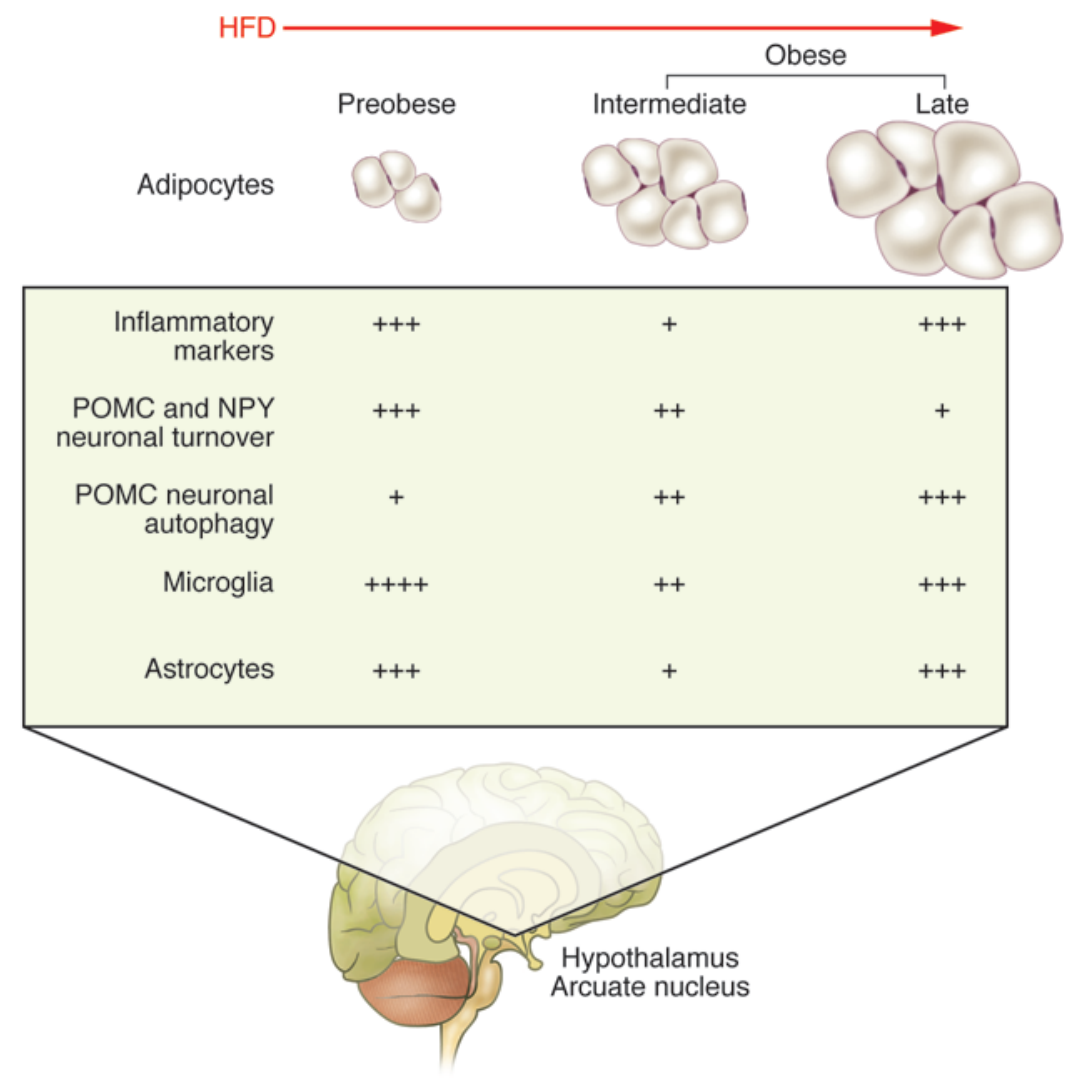

Figure 1

Schematic representation of the effects of a HFD and obesity on inflammation, neurogenesis, and gliosis in the hypothalamus. As indicated by the work of McNay et al. (12), DIO is associated with a progressive reduction in neuronal turnover in the hypothalamus. In contrast, the work of Thaler et al. (13) demonstrated that hypothalamic inflammation and gliosis displayed a biphasic pattern, with peak inflammation preceding the development of obesity (13). Plus signs indicate the degree of effect in each case.

ies have also shown an association among obesity and inflammation and autophagy (a process responsible for the degradation of damaged and dysfunctional cellular organelles and protein aggregates) in the hypothalamus (9-11). While these features suggest a potential role for hypothalamic pathology in obesity, the impact of diet and obesity on the number, structure, and temporal organization of neurons and glia in the hypothalamus and whether this has any relevance to obesity in humans is unclear. Two independent reports in this issue of the JCI $(12,13)$ have now shed some light on these matters, providing evidence that obesity alters the normal structure of the arcuate nucleus. Both studies found that the hypothalamus is dynamic in terms of neuronal turnover and glial activation and that the arcuate nucleus is particularly vulnerable to obesity. This raises the possibility that obesity may result, at least in part, from hypothalamic injury.
$63 \%$ ) after weaning, the total number of cells within the arcuate nucleus remained constant, indicating that adult neurogenesis was responsible for replacing a sizeable proportion of neurons in the arcuate nucleus. This dynamic remodeling of the arcuate nucleus included the turnover of both POMC and NPY neurons (Figure 1). Diet-induced obesity (DIO) was associated with suppression of the number of actively proliferating cells; the affected cells were then determined to be progenitor-like cells rather than neural stem-like cells. The reduction in the number of actively proliferating progenitor-like cells was linked to an increased rate of apoptosis of newly divided cells. Of note, caloric restriction in mice with DIO restored the number of actively proliferating progenitor-like cells to that seen prior to the onset of DIO.

The authors then investigated whether hypothalamic neurogenesis was similarly altered in leptin-deficient $o b / o b$ mice, which display overeating and early-onset obesity (4). They found that the number of stem cells, the small pool of cells that gives rise to actively proliferating progenitor-like cells, was drastically reduced in the hypothalamus of $o b / o b$ mice (12). Treating normal mice with leptin also seemed to increase the number of hypothalamic stem cells. This latter finding is consistent with the known stimulatory effects of leptin on neurogenesis in the hippocampus and the development of feeding circuits $(5,18)$. The results generated by McNay et al. using $o b / o b$ mice further demonstrate that obesity affects the capacity for renewal of neurons in the arcuate nucleus (12); however, it remains unclear whether hypothalamic neurogenesis is directly regulated by leptin or whether indirect effects via alterations in the levels of glucocorticoids or insulin or various other metabolic defects impact hypothalamic neurogenesis in $o b / o b$ mice (4).

\section{Feeding the flames of inflammation in obesity}

Obesity results in chronic low-grade inflammation of several organs, including the liver, muscle, and adipose tissue, as well as the hypothalamus $(8,10)$. Inflammation is thought to exacerbate metabolic abnormalities by disrupting homeostatic pathways within target tissues $(8,10)$. For example, infiltration of adipose tissue by macrophages has been linked to the development of insulin resistance $(8,10)$. Inflammation is also thought to be increased in the hypothalamus of obese 
rodents and may promote food intake and hypertension $(9-11,19)$. Although inflammation in the hypothalamus is thought to be harmful to neuronal function, little is known about the effects of a high-fat diet (HFD) on glia - nonneuronal cells that are more abundant in the central nervous system than neurons, provide support and nutrition to neurons (astrocytes), and mediate inflammation in the brain (microglia). Moreover, it is not clear how closely hypothalamic inflammation mirrors peripheral inflammation. In their study in this issue of the JCI, Thaler et al. found some interesting differences (Figure 1 and ref. 13). In liver and adipose tissue, inflammatory markers increase in number and magnitude slowly over the course of weeks to months in rats fed HFD $(8,10)$. In contrast, Thaler et al. found that inflammatory gene expression increased in the arcuate nucleus of rats within one day of initiating a HFD (13). This initial spike in inflammation in the hypothalamus occurred in the absence of weight gain and coincided with increased gliosis, the process whereby astrocytes and microglia are activated and proliferate in response to a brain insult, as measured by the expression of microglial and astrocytic genes (Figure 1). The gene expression patterns were corroborated by immunohistochemistry, which showed an increase in the number and size of reactive glia. However, the rise in inflammatory gene expression and gliosis subsided within a week and reappeared only after continued prolonged exposure to HFD. This biphasic inflammatory response to a HFD was unique to the arcuate nucleus; it was not observed in the liver, adipose tissue, and other regions of the brain. Similar patterns of inflammation and gliosis were also observed in mice fed a HFD.

What are the consequences of arcuate nucleus inflammation and gliosis in rodents fed a HFD? Thaler et al. looked for evidence of neuronal injury, particularly in POMC neurons within the arcuate nucleus (13). At seven days after a HFD was initiated, they found that many POMC neurons displayed an increase in expression of Hsp70, a chaperone protein that is induced in response to various stressors and neuronal injury. There was also evidence of increased autophagy within POMC neurons after 20 weeks on a HFD. After 8 months on a HFD, the number of POMC neurons within the arcuate nucleus was reduced by $25 \%$.
The rodent studies performed by Thaler et al. (13) provide a time line of injury to the arcuate nucleus in response to a HFD. In particular, the early induction of inflammation and reactive gliosis, together with evidence of neuronal stress within one week, suggest that the arcuate nucleus is injured acutely and that perhaps this early insult may be linked to the development of an abnormal energy balance and obesity. Thaler et al. then extended these findings by studying brain images in patients (13). They found that body mass index, a measure of fat content, was proportional to T2 signal in the hypothalamus by MRI. T2-weighted MRI is particularly sensitive for identifying brain lesions, including inflammation, gliosis, and edema. This finding raises the possibility that obesity increases hypothalamic gliosis (20), but further studies in humans are required to ascertain whether this is indeed the case and whether hypothalamic inflammation and injury occur in the arcuate nucleus, as in rodents.

\section{A long way to go}

What are the mechanisms underlying these two interesting results showing that obesity is associated with suppression of hypothalamic neurogenesis (12) and increased hypothalamic injury and gliosis (13)? With regards to the effects of obesity on hypothalamic neurogenesis (12), it appears that two distinct pools of cells, actively proliferating progenitor-like and stem-like cells, are inhibited in the hypothalamus of mice with DIO and $o b / o b$ mice, respectively. The extent to which leptin or other metabolic signals regulate neurogenesis in mice with DIO or $o b / o b$ mice is unknown. With regards to the hypothalamic inflammation (13), the data demonstrate that an initial rise in inflammatory markers is triggered by the HFD and not obesity. Rodents respond acutely to a HFD by becoming hyperphagic and consuming more calories than animals fed regular chow. It is possible that any number of factors that are altered by excessive consumption of a HFD (e.g., lipids, leptin, insulin, and ghrelin) contribute to hypothalamic inflammation, neuronal injury, and gliosis. These factors could directly impact neurons and/or glia or indirectly affect the structures and functions of these cells by changing the transport of circulating factors across the blood-brain barrier.

In summary, the studies by McNay et al. (12) and Thaler et al. (13) demonstrate that obesity is associated with profound changes in the structure of neurons and glia in the hypothalamus. Unlike the earlier hypothalamic lesion studies that were shown to cause extensive damage to neurons within and outside the hypothalamus $(1,2)$, the work of McNay et al. and Thaler et al. suggests that the consumption of a HFD results in acute hypothalamic injury, followed by chronic changes in the structure and organization of neurons and glia. However, there is no evidence that these changes in hypothalamic structure are responsible for the onset and progression of obesity and associated metabolic disorders. Understanding how the intake of a HFD leads to injury and remodeling of the hypothalamus and whether this affects the development of obesity may provide novel insights into the pathogenesis of obesity. Furthermore, it is crucial to investigate whether hypothalamic neurogenesis, inflammation, and gliosis are reversible after weight loss in rodents and humans; whether the dynamics of neurons and glia are causally related to the failure to sustain weight reduction after dieting; and whether the structural changes in the hypothalamus could be targeted in the treatment of obesity and related diseases.

\section{Acknowledgments}

Edward B. Lee is supported by NIH grant K08AG039510. Rexford S. Ahima is supported by NIH grants R01DK062348, P01DK049210, and P30-DK19525.

Address correspondence to: Edward B. Lee, University of Pennsylvania, 605B Stellar Chance Laboratories, 422 Curie Blvd., Philadelphia, Pennsylvania 19104, USA. Phone: 215.898.0908; Fax: 215.898.9989; E-mail: edward.lee@uphs.upenn.edu. Or to: Rexford S. Ahima, University of Pennsylvania, 12-104 Translational Research Center, 3400 Civic Center Blvd., Building 421, Philadelphia, Pennsylvania 19104, USA. Phone: 215.573.1872; Fax: 215.898.5408; E-mail: ahima@mail.med.upenn.edu.

1. Stellar E. The physiology of motivation. Psychol Rev. 1954;61(1):5-22.

2. Tepperman J, Brobeck JR, Long CN. The effects of hypothalamic hyperphagia and of alterations in feeding habits on the metabolism of the albino rat. Yale J Biol Med. 1943;15(6):855-874.

3. Elmquist JK, Elias CF, Saper CB. From lesions to leptin: hypothalamic control of food intake and body weight. Neuron. 1999;22(2):221-232.

4. Ahima RS, Bjorbaek C, Osei S, Flier JS. Regulation of neuronal and glial proteins by leptin: implications for brain development. Endocrinology. 1999;140(6):2755-2762.

5. Bouret SG, Draper SJ, Simerly RB. Trophic action of leptin on hypothalamic neurons that regulate 
feeding. Science. 2004;304(5667):108-110.

6. Bouret SG, Gorski JN, Patterson CM, Chen S, Levin BE, Simerly RB. Hypothalamic neural projections are permanently disrupted in diet-induced obese rats. Cell Metab. 2008;7(2):179-185.

7. Dietrich MO, Horvath TL. Synaptic plasticity of feeding circuits: hormones and hysteresis. Cell. 2011; 146(6):863-865

8. Sun K, Kusminski CM, Scherer PE. Adipose tissue remodeling and obesity. J Clin Invest. 2011; 121(6):2094-2101.

9. Kaushik S, et al. Autophagy in hypothalamic AgRP neurons regulates food intake and energy balance. Cell Metab. 2011;14(2):173-183.

10. Lumeng CN, Saltiel AR. Inflammatory links between obesity and metabolic disease. J Clin Invest. 2011; 121(6):2111-2117.

11. Meng Q, Cai D. Defective hypothalamic autophagy directs the central pathogenesis of obesity via the IkappaB kinase beta (IKKbeta)/NF-kappaB pathway. J Biol Chem. 2011;286(37):32324-32332.

12. McNay DEG, Briançon N, Kokoeva MV, MaratosFlier E, Flier JS. Remodeling of the arcuate nucleus energy-balance circuit is inhibited in obese mice. J Clin Invest. 2012;122(1):142-152.

13. Thaler JP, et al. Obesity is associated with hypothalamic injury in rodents and humans. J Clin Invest. 2012;122(1):153-162.

14. Ettinger MP, et al. Recombinant variant of ciliary neurotrophic factor for weight loss in obese adults: a randomized, dose-ranging study. JAMA. 2003;289(14):1826-1832.

15. Watt MJ, et al. CNTF reverses obesity-induced insulin resistance by activating skeletal muscle AMPK. Nature Med. 2006;12(5):541-548.

16. Kokoeva MV, Yin H, Flier JS. Neurogenesis in the hypothalamus of adult mice: potential role in energy balance. Science. 2005;310(5748):679-683.

17. Kokoeva MV, Yin H, Flier JS. Evidence for constitutive neural cell proliferation in the adult murine hypothalamus. J Comp Neurol. 2007; 505(2):209-220.

18. Shanley LJ, Irving AJ, Rae MG, Ashford ML, Harvey J. Leptin inhibits rat hippocampal neurons via activation of large conductance calcium-activated K+ channels. Nat Neurosci. 2002;5(4):299-300.

19. Purkayastha S, Zhang G, Cai D. Uncoupling the mechanisms of obesity and hypertension by targeting hypothalamic IKK-beta and NF-kappaB. Nat Med. 2011;17(7):883-887.

20. Freeman JL, et al. MR imaging and spectroscopic study of epileptogenic hypothalamic hamartomas: analysis of 72 cases. AJNR Am J Neuroradiol. 2004; 25(3):450-462.

\title{
Rare serotype adenoviral vectors for HIV vaccine development
}

\author{
Nelson L. Michael
}

US Military HIV Research Program, Walter Reed Army Institute of Research, Bethesda, Maryland, USA.

\begin{abstract}
Human adenoviral vectors are being developed for use in candidate vaccines for HIV-1 and other pathogens. However, this approach suffered a setback when an HIV-1 vaccine using an adenovirus type 5 (Ad5) vector failed to reduce, and might even have increased, the rate of HIV infection in men who were uncircumcised and who had preexisting antibodies specific for Ad5. This increased interest in the evaluation of serologically distinct adenoviral vectors. In this issue of the JCI, Frahm and coworkers report evidence that preexisting cellular immune responses directed toward Ad5 reduce the immunogenicity of antigens expressed in Ad5-vectored vaccines and have cross-reacting potential with non-Ad5 adenoviral vectors. The implications of this observation need to be carefully evaluated in future clinical trials of all serotypes of adenovirus-vectored vaccines.
\end{abstract}

The joint United Nations Program on HIV/ AIDS (UNAIDS) estimates that more than 33 million people were living with HIV at the end of 2009 (1). As witnessed by a series of watershed results in the HIV prevention field of late, some progress has been made toward tackling this pandemic. Adult male circumcision (2-4), antiretroviral-based vaginal microbicides (5), preexposure prophylaxis (6), and antiretroviral therapy as a means of prevention (7) have shown varying degrees of efficacy, from modest to potent. These results have together energized the HIV prevention field and have provided a growing number of tools that could be brought to bear in global HIV disease control. However, as all of these approaches

Conflict of interest: The author has declared that no conflict of interest exists.

Citation for this article: J Clin Invest. 2012; 122(1):25-27. doi:10.1172/JCI60988. pose significant challenges for deployment as public health tools, a preventive HIV vaccine, development of which has thus far been elusive despite intensive research efforts, remains a critical goal (8).

\section{HIV vaccine approaches}

There are three main types of vaccine being developed for the prevention of HIV infection: subunit vaccines, recombinant virus-vectored vaccines, and DNA vaccines. Three of the most significant clinical trials conducted thus far tested the efficacy of different combinations of the first two types of vaccine (9-11). When the first vaccine type was tested in two phase III clinical trials, it was disappointingly found that vaccination with repeated doses of gp120 protein subunit vaccines (AIDSVAX B/B' and $\mathrm{B} / \mathrm{E}$ ) failed to protect either men who have sex with men (MSM) (10) or injection drug users (11) from HIV infection. Further disappointment came in the form of the results of the phase III Step study (9), which showed that three injections of a Merckdeveloped adenovirus serotype 5 (Ad5) vector containing gag/pol/nef HIV-1 gene inserts (referred to as the MRKAd5/HIV-1 vaccine) also failed to protect MSM. In contrast to these results, the RV144 trial demonstrated that an HIV vaccine might be possible (12). The approach tested in this trial was to first prime participants with four injections of a canarypox vector containing gag/pro/gp120/ gp41 HIV-1 gene inserts (ALVAC-HIV) and then boost with two injections of a gp120 protein subunit vaccine (AIDSVAX-B/E), and it reduced the rate of HIV infection in a low-incidence population of Thais with heterosexual HIV transmission risk.

The Step study raised the added concern that, following vaccination, uncircumcised MSM with naturally acquired immunity to Ad5, in the guise of preexisting Ad5-specific neutralizing antibodies (nAbs), appeared to experience a transient period of increased risk of infection with HIV (13). These concerns led to the cancellation of a planned phase IIB clinical trial of a related Ad5-vectored gag/pol/env vaccine, which was to be used together with a DNA vaccine prime, in diverse risk groups and the generation of a new, smaller efficacy study focusing on circumcised MSM with no serologic evidence of previous Ad5 exposure (14). Additional research has suggested that the immunoge- 